\title{
Intrapericardial bleomycin for the management of cardiac tamponade secondary to malignant pericardial effusion
}

\author{
M C Cormican, C R Nyman
}

\begin{abstract}
A patient with malignant pericardial effusion with cardiac tamponade was treated by the instillation of intrapericardial bleomycin. The tumour deposits elsewhere in the body continued to progress and eventually killed the patient, but drainage and one dose of intrapericardial bleomycin prevented further episodes of pericardial effusion.
\end{abstract}

Malignant pericardial effusions are said to be responsible for one third of cases of cardiac tamponade. ${ }^{1}$ This condition often presents as a medical emergency, though it can present gradually with progression of signs over several hours, or indeed days. The situation is usually life threatening and prompt adequate treatment is essential. Percutaneous drainage of the pericardial effusion produces instant relief of the signs and symptoms and instillation of various substances, including antibiotics (tetracycline) and chemotherapeutic agents (nitrogen mustard, thiotepa, mepacrine hydrochloride) have been used with varying degrees of success. Bleomycin is less often used in this condition but is efficacious. We describe a patient in whom intrapericardial bleomycin provided useful palliation, although the tumour was unresponsive to systemic chemotherapy.

\section{Case report}

A 53 year old man was admitted to the hospital with a seven day history of dyspnoea on exertion and left sided chest pain. Examination showed a regular tachycardia of 120 per minute, pulsus paradoxus, a raised jugular venous pressure, a blood pressure of 110 / $70 \mathrm{~mm} \mathrm{Hg}$, and an enlarged lymph gland in the left posterior triangle of his neck. There was a moderate left sided effusion. Chest $x$ ray showed a large heart with clear margins, a mass eroding the right third rib, and confirmed the left sided pleural effusion (fig la). The echocardiogram confirmed a large pericardial effusion (fig 1b). Cytological examination of the pleural fluid showed adenocarcinoma cells. Over the next 24 hours the patient's condition deteriorated with symptoms of dizziness and increasing breathlessness, a rise in the pulse rate to 150 per minute, and a fall in systemic blood pressure to $100 /$ $60 \mathrm{~mm} \mathrm{Hg}$. At this stage we placed a percutaneous catheter in the pericardial sac using a Seldinger technique and drained $800 \mathrm{ml}$ of heavily bloodstained fluid. This produced prompt relief of the symptoms and improved his signs. Adenocarcinoma cells were found in the pericardial fluid.

Free drainage of the pericardial space via the catheter continued for four days, then $5 \mathrm{mg}$ of bleomycin in $20 \mathrm{ml}$ of water was instilled into the pericardial sac. The catheter
Department of Cardiorespiratory Medicine, Pilgrim Hospital, Boston, Lincolnshire M C Cormican C R Nyman

Correspondence to Dr C R Nyman, Department of Cardiorespiratory Medicine, Pilgrim Hospital, Sibsey Road, Boston Lincolnshire PE21 9QS.
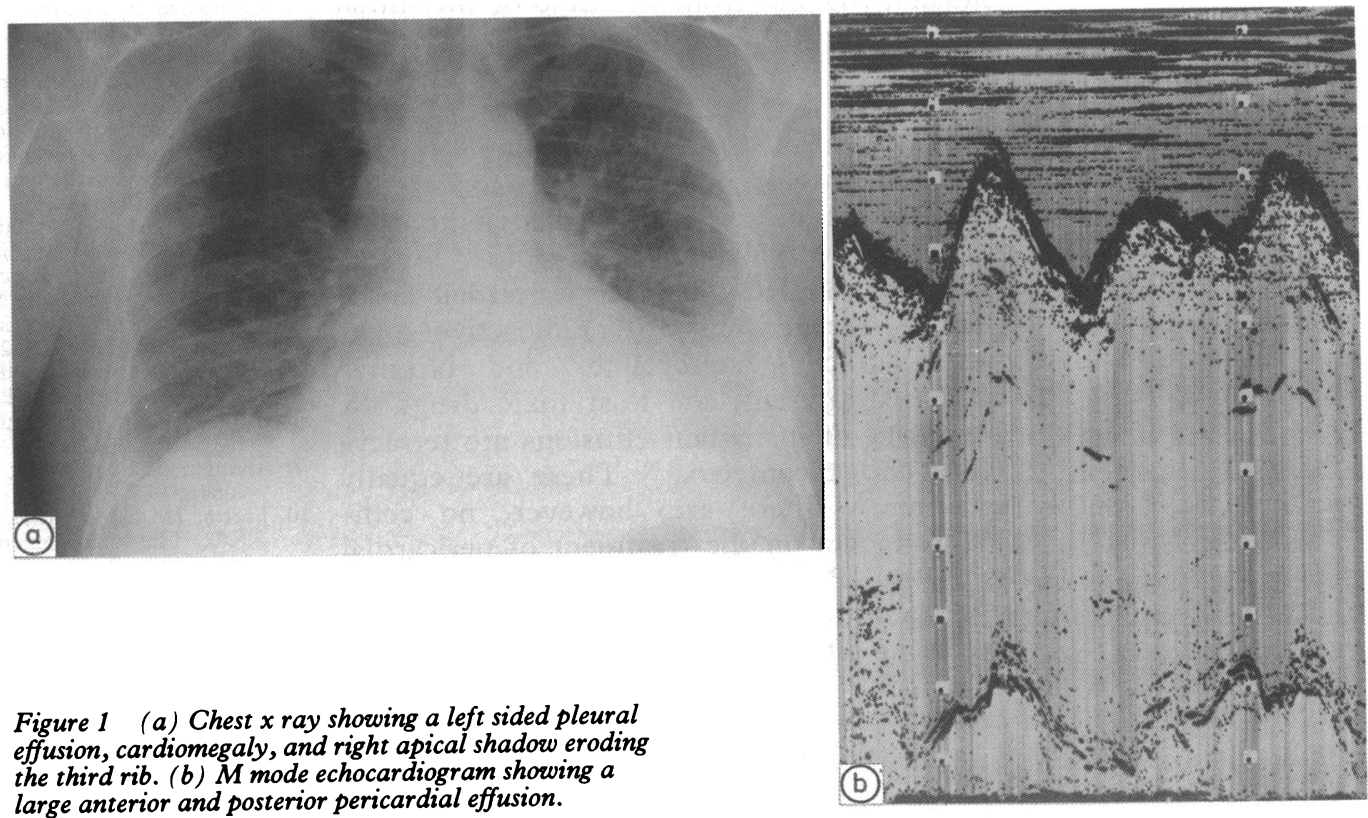

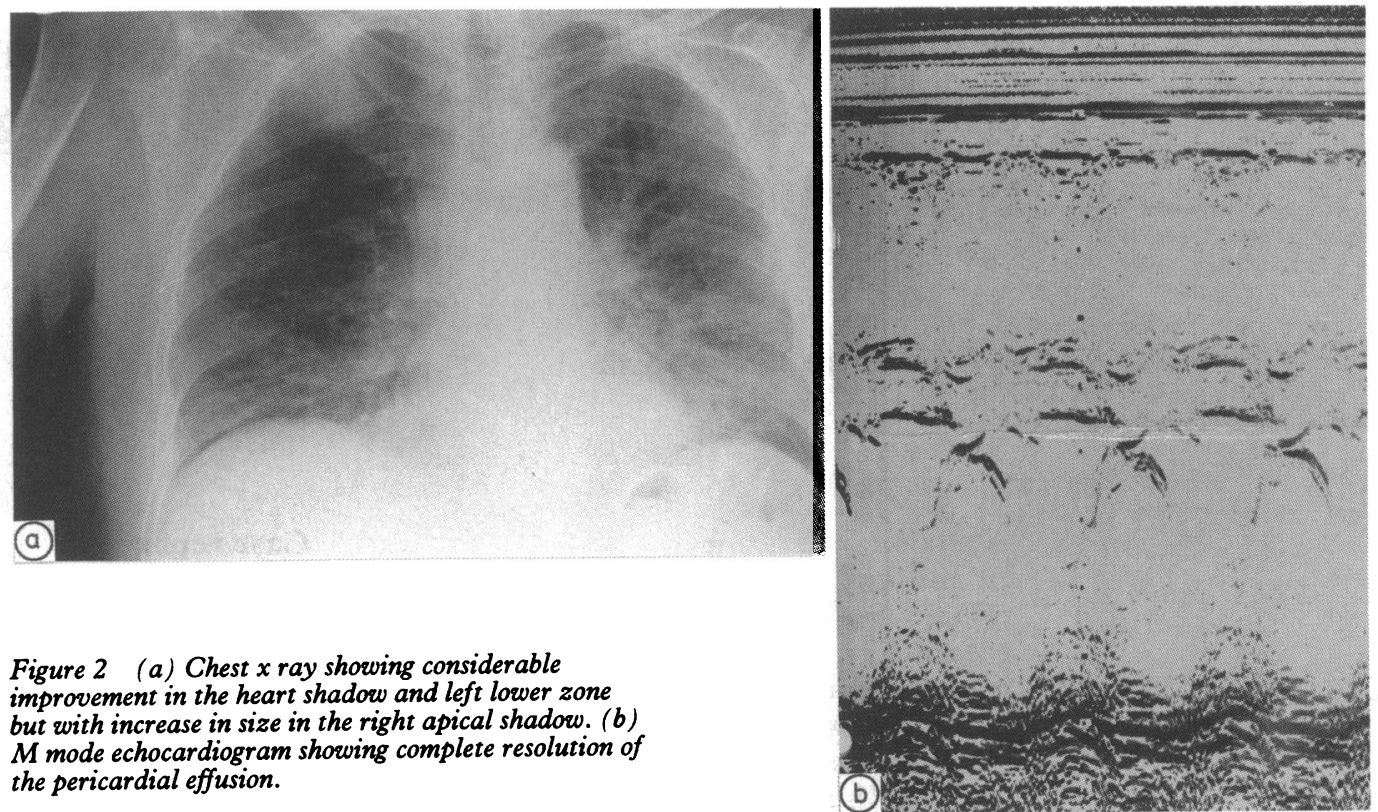

Figure 2 (a) Chest $x$ ray showing considerable improvement in the heart shadow and left lower zone but with increase in size in the right apical shadow. (b) $M$ mode echocardiogram showing complete resolution of the pericardial effusion.

was then clamped and removed 24 hours later. No adverse effects were seen with this procedure, either during the acute period or afterwards. However, the chest pain that was present on admission persisted. Despite an extensive search for the underlying primary site, we found no evidence of tumours in the kidney, gastrointestinal system, bronchus, or pancreas.

We thought that the tumour was probably pulmonary in origin. Despite the low response rate of lung adenocarcinomas to chemotherapy, we believed that it was appropriate to give this man a trial of combination chemotherapy. He was started on monthly courses of 24 hour treatment with ifosfamide, etoposide, vincristine, and mesna. Despite continued tumour growth at other sites (including the neck, a right sided mass on chest $x$ ray (fig $2 a$ ), recurrence of pleural effusion, and subsequently cerebral metastases), there was no clinical, radiological, or echocardiographic evidence of recurrence of the pericardial effusion (fig 2b) from the time of the initial diagnosis up to death 65 days after presentation.

\section{Discussion}

It is better to use conservative measures rather than operation to treat cardiac tamponade secondary to malignant pericarditis. ${ }^{2}$ Agents that are instilled into the pericardial space include nitrogen mustard, ${ }^{2}$ radioactive gold, ${ }^{3}$ 5-fluorouracil, ${ }^{4}$ tetracycline, ${ }^{5}$ and bleomycin. $^{6-8}$ At present, the least toxic drugs for treatment of malignant effusions are tetracycline and bleomycin..$^{10}$ These are equally effective. ${ }^{11}$ There are, however, no comparative data on the treatment of pericardial effusion. Published reports suggest that pyrexia and pain may be more common after the use of tetracycline. ${ }^{69}$
Bleomycine is toxic at doses of $40 \mathrm{mg} / \mathrm{m}^{2}$ much in excess of that required for control of pericardial effusion. We know of eight reported cases of the use of intrapericardial instillation of bleomycin. In six there was no recurrence of effusion up to the time of death and one patient survived for 434 days. The effusion recurred 14 days after treatment in one patient and at 2.5 months in another. No recurrence of effusion was noted after pericardial instillation of tetracycline in six patients. ${ }^{6}$ Tetracycline and bleomycin seem to be equally effective but we believe that more information on treatment failures and adverse reactions is required to clarify the role of these agents in the treatment of this uncommon disorder.

1 Guberman BA, Fowler NO, Engel PJ, Gueron M, Allen JM. Cardiac tamponade in medical patients. Circulation Cardiac tampo

2 Smith FE, Lane M, Hudgins PT. Conservative management of malignant pericardial effusion. Cancer 1974;33:47-57.

3 Castleman B, McNeely BV. Case records of the Massachusetts General Hospital. $N$ Engl J Med 1964;271: $1160-5$.

4 Suhrland LG, Weisberger AS. Intracavitary 5-fluorouracil in malignant effusions. Arch Intern Med 1965;116:431-3. Davis S, Sharma SM, Blumberg ED, Kim CS. Intrapericardial tetraclycine for the management of cardiac tamponade secondary to malignant pericardial effusion. $N \mathrm{Engl} \mathrm{J} \mathrm{Med}$ 1978;299:1113-4.

6 Wei JY, Taylor GJ, Achuff SC. Recurrent cardiac tamponade and large pericardial effusions: management with an indwelling pericardial catheter. Am $J$ Cardiol 1978;42:28.

7 Volckaert A, Van Belle S, Taeymans Y. Malignant pericardial effusion treated by drainage and intracavitary cardial effusion treated by drainage and intracavitary Congress, Budapest 1986.

8 Maher ER, Buckman R. Intrapericardial instillation of bleomycin in malignant pericardial effusion. Am Heart $J$ 1986;III:613-4.

9 Anonymous. Treatment of malignant effusions. Lancet 1981;1:198.

10 Trotter JM, Stuart JEB, McBeath F, McVie JG, Calman KC. The management of malignant effusions with bleomy $B r J$ Cancer 1979;40:316.

1 Gupta N, Opfell RW, Padova J, Margileth D, Souadjian J. Intrapleural bleomycin versus tetracycline for control of malignant pleural effusion. A randomised study [Abstract C-189]. Proc Am Assoc Clin Oncol 1980:21. 